\title{
$\mu$-Stability of Impulsive Neural Networks with Unbounded Time-Varying Delays and Continuously Distributed Delays
}

\author{
Lizi Yin ${ }^{1,2}$ and Xilin Fu ${ }^{3}$ \\ ${ }^{1}$ School of Management and Economics, Shandong Normal University, Jinan 250014, China \\ ${ }^{2}$ School of Science, University of Jinan, Jinan 250022, China \\ ${ }^{3}$ School of Mathematical Sciences, Shandong Normal University, Jinan 250014, China \\ Correspondence should be addressed to Lizi Yin, ss_yinlz@ujn.edu.cn
}

Received 13 November 2010; Revised 19 February 2011; Accepted 3 March 2011

Academic Editor: Jin Liang

Copyright (C) 2011 L. Yin and X. Fu. This is an open access article distributed under the Creative Commons Attribution License, which permits unrestricted use, distribution, and reproduction in any medium, provided the original work is properly cited.

This paper is concerned with the problem of $\mu$-stability of impulsive neural systems with unbounded time-varying delays and continuously distributed delays. Some $\mu$-stability criteria are derived by using the Lyapunov-Krasovskii functional method. Those criteria are expressed in the form of linear matrix inequalities (LMIs), and they can easily be checked. A numerical example is provided to demonstrate the effectiveness of the obtained results.

\section{Introduction}

In recent years, the dynamics of neural networks have been extensively studied because of their application in many areas, such as associative memory, pattern recognition, and optimization [1-4]. Many researchers have a lot of contributions to these subjects. Stability is a basic knowledge for dynamical systems and is useful to the real-life systems. The time delays happen frequently in various engineering, biological, and economical systems, and they may cause instability and poor performance of practical systems. Therefore, the stability analysis for neural networks with time-delay has attracted a large amount of research interest, and many sufficient conditions have been proposed to guarantee the stability of neural networks with various type of time delays, see for example [5-20] and the references therein. However, most of the results are obtained based on the assumption that the time delay is bounded. As we know, time delays occur and vary frequently and irregularly in many engineering systems, and sometimes they depend on the histories heavily and may be unbounded [21, 22]. In such case, those existing results in [5-20] are all invalid. 
How to guarantee the desirable stability if the time delays are unbounded? Recently, Chen et al. $[23,24]$ proposed a new concept of $\mu$-stability and established some sufficient conditions to guarantee the global $\mu$-stability of delayed neural networks with or without uncertainties via different approaches. Those results can be applied to neural networks with unbounded time-varying delays. Moreover, few results have been reported in the literature concerning the problem of $\mu$-stability of impulsive neural networks with unbounded timevarying delays and continuously distributed delays. As we know, the impulse phenomenon as well as time delays are ubiquitous in the real world [25-27]. The systems with impulses and time delays can describe the real world well and truly. This inspire our interests.

In this paper, we investigate the problem of $\mu$-stability for a class of impulsive neural networks with unbounded time-varying delays and continuously distributed delays. Based on Lyapunov-Krasovskii functional and some analysis techniques, several sufficient conditions that ensure the $\mu$-stability of the addressed systems are derived in terms of LMIs, which can easily be checked by resorting to available software packages. The organization of this paper is as follows. The problems investigated in the paper are formulated, and some preliminaries are presented, in Section 2. In Section 3, we state and prove our main results. Then, a numerical example is given to demonstrate the effectiveness of the obtained results in Section 4. Finally, concluding remarks are made in Section 5.

\section{Preliminaries}

\section{Notations}

Let $\mathbb{R}$ denote the set of real numbers, $\mathbb{Z}_{+}$denote the set of positive integers, and $\mathbb{R}^{n}$ denote the $n$-dimensional real spaces equipped with the Euclidean norm $|\cdot|$. Let $\mathscr{A} \geq 0$ or $\mathscr{A} \leq 0$ denote that the matrix $\mathscr{A}$ is a symmetric and positive semidefinite or negative semidefinite matrix. The notations $\mathscr{A}^{T}$ and $\mathcal{A}^{-1}$ mean the transpose of $\mathcal{A}$ and the inverse of a square matrix. $\lambda_{\max }(\mathcal{A})$ or $\lambda_{\min }(\mathcal{A})$ denote the maximum eigenvalue or the minimum eigenvalue of matrix $\mathcal{A}$. I denotes the identity matrix with appropriate dimensions and $\Lambda=\{1,2, \ldots, n\}$. In addition, the notation $\star$ always denotes the symmetric block in one symmetric matrix.

Consider the following impulsive neural networks with time delays:

$$
\begin{aligned}
& \dot{x}(t)=-C x(t)+A f(x(t))+B f(x(t-\tau(t))) \\
&+W \int_{0}^{\infty} h(s) f(x(t-s)) d s+J, \quad t \neq t_{k}, \quad t>0, \\
& \Delta x\left(t_{k}\right)=x\left(t_{k}\right)-x\left(t_{k}^{-}\right)=J_{k}\left(x\left(t_{k}^{-}\right)\right), \quad k \in \mathbb{Z}_{+},
\end{aligned}
$$

where the impulse times $t_{k}$ satisfy $0=t_{0}<t_{1}<\cdots<t_{k}<\cdots, \lim _{k \rightarrow \infty} t_{k}=+\infty ; x(t)=$ $\left(x_{1}(t), \ldots, x_{n}(t)\right)^{T}$ is the neuron state vector of the neural network; $C=\operatorname{diag}\left(c_{1}, \ldots, c_{n}\right)$ is a diagonal matrix with $c_{i}>0, i=1, \ldots, n ; A, B, W$ are the connection weight matrix, the delayed weight matrix, and the distributively delayed connection weight matrix, respectively; $J$ is an input constant vector; $\tau(t)$ is the transmission delay of the neural networks; $f(x(\cdot))=\left(f_{1}\left(x_{1}(\cdot)\right), \ldots, f_{n}\left(x_{n}(\cdot)\right)\right)^{T}$ represents the neuron activation function; $h(\cdot)=\operatorname{diag}\left(h_{1}(\cdot), \ldots, h_{n}(\cdot)\right)$ is the delay kernel function and $J_{k}$ is the impulsive function. 
Throughout this paper, the following assumptions are needed.

$\left(\mathrm{H}_{1}\right)$ The neuron activation functions $f_{j}(\cdot), j \in \Lambda$, are bounded and satisfy

$$
\delta_{j}^{-} \leq \frac{f_{j}(u)-f_{j}(v)}{u-v} \leq \delta_{j}^{+}, \quad j \in \Lambda,
$$

for any $u, v \in \mathbb{R}, u \neq v$. Moreover, we define

$$
\Sigma_{1}=\operatorname{diag}\left(\delta_{1}^{-} \delta_{1}^{+}, \ldots, \delta_{n}^{-} \delta_{n}^{+}\right), \quad \Sigma_{2}=\operatorname{diag}\left(\frac{\delta_{1}^{-}+\delta_{1}^{+}}{2}, \ldots, \frac{\delta_{n}^{-}+\delta_{n}^{+}}{2}\right),
$$

where $\delta_{j}^{-}, \delta_{j}^{+}, j \in \Lambda$ are some real constants and they may be positive, zero, or negative.

$\left(\mathrm{H}_{2}\right)$ The delay kernels $h_{j}, j \in \Lambda$, are some real value nonnegative continuous functions defined in $[0, \infty)$ and satisfy

$$
\int_{0}^{\infty} h_{j}(s) d s=1
$$

$\left(\mathrm{H}_{3}\right) \tau(t)$ is a nonnegative and continuously differentiable time-varying delay and satisfies $\dot{\tau}(t) \leq \rho<1$, where $\rho$ is a positive constant.

If the function $f_{j}$ satisfies the hypotheses $\left(\mathrm{H}_{1}\right)$ above, there exists an equilibrium point for system (2.1), see [28]. Assume that $x^{*}=\left(x_{1}^{*}, \ldots, x_{n}^{*}\right)^{T}$ is an equilibrium of system (2.1) and the impulsive function in system (2.1) characterized by $J_{k}\left(x\left(t_{k}^{-}\right)\right)=-D_{k}\left(x\left(t_{k}^{-}\right)-x^{*}\right)$, where $D_{k}$ is a real matrix. Then, one can derive from (2.1) that the transformation $y=x-x^{*}$ transforms system (2.1) into the following system:

$$
\begin{aligned}
\dot{y}(t)= & -C y(t)+A g(y(t))+B g(y(t-\tau(t))) \\
& +W \int_{0}^{\infty} h(s) g(y(t-s)) d s, \quad t \neq t_{k}, t>0, \\
\Delta y\left(t_{k}\right)= & y\left(t_{k}\right)-y\left(t_{k}^{-}\right)=-D_{k} y\left(t_{k}^{-}\right), \quad k \in \mathbb{Z}_{+},
\end{aligned}
$$

where $g(y(\cdot))=f\left(y(\cdot)+x^{*}\right)-f\left(x^{*}\right)$.

Obviously, the $\mu$-stability analysis of the equilibrium point $x^{*}$ of system (2.1) can be transformed to the $\mu$-stability analysis of the trivial solution $y=0$ of system (2.5). For completeness, we first give the following definition and lemmas.

Definition 2.1 (see [23]). Suppose that $\mu(t)$ is a nonnegative continuous function and satisfies $\mu(t) \rightarrow \infty$ as $t \rightarrow \infty$. If there exists a scalar $M>0$ such that

$$
\|x\| \leq \frac{M}{\mu(t)}, \quad t \geq 0
$$

then the system (2.1) is said to be $\mu$-stable. 
Obviously, the definition of $\mu$-stable includes the global asymptotical and the global exponential stability.

Lemma 2.2 (see [29]). For a given matrix

$$
S=\left(\begin{array}{ll}
S_{11} & S_{12} \\
S_{21} & S_{22}
\end{array}\right)>0,
$$

where $S_{11}^{T}=S_{11}, S_{22}^{T}=S_{22}$, is equivalent to any one of the following conditions:

(1) $S_{22}>0, S_{11}-S_{12} S_{22}^{-1} S_{12}^{T}>0$;

(2) $S_{11}>0, S_{22}-S_{12}^{T} S_{11}^{-1} S_{12}>0$.

\section{Main Results}

Theorem 3.1. Assume that assumptions $\left(H_{1}\right),\left(H_{2}\right)$, and $\left(H_{3}\right)$ hold. Then, the zero solution of system (2.5) is $\mu$-stable if there exist some constants $\beta_{1} \geq 0, \beta_{2}>0, \beta_{3}>0$, two $n \times n$ matrices $P>0, Q>0$, two diagonal positive definite $n \times n$ matrices $M=\operatorname{diag}\left(m_{1}, \ldots, m_{n}\right), U$, a nonnegative continuous differential function $\mu(t)$ defined on $[0, \infty)$, and a constant $T>0$ such that, for $t \geq T$

$$
\frac{\dot{\mu}(t)}{\mu(t)} \leq \beta_{1}, \quad \frac{\mu(t-\tau(t))}{\mu(t)} \geq \beta_{2}, \quad \frac{\int_{0}^{\infty} h_{j}(s) \mu(s+t) d s}{\mu(t)} \leq \beta_{3}, \quad j \in \Lambda,
$$

and the following LMIs hold:

$$
\begin{gathered}
{\left[\begin{array}{cccc}
\Sigma & P A+U \Sigma_{2} & P B & P W \\
\star & Q+N-U & 0 & 0 \\
\star & \star & -\beta_{2} Q(1-\rho) & 0 \\
\star & \star & \star & -M
\end{array}\right] \leq 0,} \\
{\left[\begin{array}{cc}
P & \left(I-D_{k}\right) P \\
\star & P
\end{array}\right] \geq 0,}
\end{gathered}
$$

where $\Sigma=\beta_{1} P-P C-C P-U \Sigma_{1}, N=\operatorname{diag}\left(m_{1} \beta_{3}, \ldots, m_{n} \beta_{3}\right)$.

Proof. Consider the Lyapunov-Krasovskii functional:

$$
\begin{aligned}
V(t)= & \mu(t) y^{T}(t) P y(t)+\int_{t-\tau(t)}^{t} \mu(s) g^{T}(y(s)) Q g(y(s)) d s \\
& +\sum_{j=1}^{n} m_{j} \int_{0}^{\infty} h_{j}(\sigma) \int_{t-\sigma}^{t} \mu(s+\sigma) g_{j}^{2}\left(y_{j}(s)\right) d s d \sigma .
\end{aligned}
$$


The time derivative of $V$ along the trajectories of system (2.5) can be derived as

$$
\begin{aligned}
D^{+} V= & \dot{\mu}(t) y^{T}(t) P y(t)+2 \mu(t) y^{T}(t) P \dot{y}(t)+\mu(t) g^{T}(y(t)) Q g(y(t)) \\
& -\mu(t-\tau(t)) g^{T}(y(t-\tau(t))) Q g(y(t-\tau(t)))[1-\dot{\tau}(t)] \\
& +\sum_{j=1}^{n} m_{j} g_{j}^{2}\left(y_{j}(t)\right) \int_{0}^{\infty} \mu(\sigma+t) h_{j}(\sigma) d \sigma \\
& -\mu(t) \sum_{j=1}^{\infty} m_{j} \int_{0}^{\infty} h_{j}(\sigma) g_{j}^{2}\left(y_{j}(t-\sigma)\right) d \sigma \leq \dot{\mu}(t) y^{T}(t) P y(t)+2 \mu(t) y^{T}(t) P \\
& \times\left[-C y(t)+A g(y(t))+B g(y(t-\tau(t)))+W \int_{0}^{\infty} h(s) g(y(t-s)) d s\right] \\
& +\mu(t) g^{T}(y(t)) Q g(y(t)) \\
& -\mu(t-\tau(t)) g^{T}(y(t-\tau(t))) Q g(y(t-\tau(t)))[1-\rho] \\
& +\mu(t) \sum_{j=1}^{n} m_{j} g_{j}^{2}\left(y_{j}(t)\right) \frac{\int_{0}^{\infty} \mu(\sigma+t) h_{j}(\sigma) d \sigma}{\mu(t)} \\
& -\mu(t) \sum_{j=1}^{n} m_{j} \int_{0}^{\infty} h_{j}(\sigma) g_{j}^{2}\left(y_{j}(t-\sigma)\right) d \sigma .
\end{aligned}
$$

It follows from the assumption (3.1) that

$$
\sum_{j=1}^{n} m_{j} g_{j}^{2}\left(y_{j}(t)\right) \frac{\int_{0}^{\infty} \mu(\sigma+t) h_{j}(\sigma) d \sigma}{\mu(t)} \leq \sum_{j=1}^{n} m_{j} \beta_{3} g_{j}^{2}\left(y_{j}(t)\right)=g^{T}(y(t)) N g(y(t))
$$

We use the assumption $\left(\mathrm{H}_{2}\right)$ and Cauchy's inequality $\left(\int p(s) q(s)\right)^{2} \leq\left(\int p^{2}(s) d s\right)\left(\int q^{2}(s) d s\right)$ and get

$$
\begin{aligned}
\sum_{j=1}^{n} m_{j} \int_{0}^{\infty} h_{j}(\sigma) g_{j}^{2}\left(y_{j}(t-\sigma)\right) d \sigma= & \sum_{j=1}^{n} m_{j} \int_{0}^{\infty} h_{j}(\sigma) d \sigma \int_{0}^{\infty} h_{j}(\sigma) g_{j}^{2}\left(y_{j}(t-\sigma)\right) d \sigma \\
\geq & \sum_{j=1}^{n} m_{j}\left[\int_{0}^{\infty} h_{j}(\sigma) g_{j}\left(y_{j}(t-\sigma)\right) d \sigma\right]^{2} \\
= & {\left[\int_{0}^{\infty} h(\sigma) g(y(t-\sigma)) d \sigma\right]^{T} } \\
& \times M\left[\int_{0}^{\infty} h(\sigma) g(y(t-\sigma)) d \sigma\right] .
\end{aligned}
$$


Note that, for any $n \times n$ diagonal matrix $U>0$ it follows that

$$
\mu(t)\left(\begin{array}{c}
y(t) \\
g(y(t))
\end{array}\right)^{T}\left(\begin{array}{cc}
-U \Sigma_{1} & U \Sigma_{2} \\
\star & -U
\end{array}\right)\left(\begin{array}{c}
y(t) \\
g(y(t))
\end{array}\right) \geq 0
$$

Substituting (3.5), (3.6) and (3.7), to (3.4), we get, for $t \geq T$,

$$
\begin{aligned}
D^{+} V \leq & \mu(t) y^{T}(t)\left[\frac{\dot{\mu}(t)}{\mu(t)} P-P C-C P-U \Sigma_{1}\right] y(t) \\
& +2 \mu(t) y^{T}(t)\left[P A+U \Sigma_{2}\right] g(y(t))+2 \mu(t) y^{T}(t) P B g(y(t-\tau(t))) \\
& +2 \mu(t) y^{T}(t) P W \int_{0}^{\infty} h(\sigma) g(y(t-\sigma)) d \sigma \\
& -\mu(t-\tau(t)) g^{T}(y(t-\tau(t))) Q g(y(t-\tau(t)))[1-\rho] \\
& +\mu(t) g^{T}(y(t))[N+Q-U] g(y(t)) \\
& -\mu(t)\left[\int_{0}^{\infty} h(\sigma) g(y(t-\sigma)) d \sigma\right]^{T} M\left[\int_{0}^{\infty} h(\sigma) g(y(t-\sigma)) d \sigma\right] \\
& {\left[\begin{array}{c}
y(t) \\
y(t) \cdot[y(t)) \\
g(y(t-\tau(t))) \\
\int_{0}^{\infty} h(s) g(y(t-s)) d s
\end{array}\right]^{T}\left[\begin{array}{c}
g(y(t)) \\
g(s) g(y(t-s)) d s] \\
\left.\left.\int_{0}^{\infty} h(t)\right)\right)
\end{array}\right] }
\end{aligned}
$$

where

$$
\Xi=\left[\begin{array}{cccc}
\Sigma & P A+U \Sigma_{2} & P B & P W \\
\star & Q+N-U & 0 & 0 \\
\star & \star & -\beta_{2} Q(1-\rho) & 0 \\
\star & \star & \star & -M
\end{array}\right] .
$$

So, by assumption (3.2) and (3.8), we have

$$
D^{+} V \leq 0 \quad \text { for } t \in\left[t_{k-1}, t_{k}\right) \cap[T, \infty), k \in \mathbb{Z}_{+} .
$$


In addition, we note that

$$
\begin{aligned}
{\left[\begin{array}{cc}
P & \left(I-D_{k}\right) P \\
\star & P
\end{array}\right] } & \geq 0 \\
& \Longleftrightarrow\left[\begin{array}{cc}
I & 0 \\
0 & P^{-1}
\end{array}\right]\left[\begin{array}{cc}
P & \left(I-D_{k}\right) P \\
\star & P
\end{array}\right] \quad\left[\begin{array}{cc}
I & 0 \\
0 & P^{-1}
\end{array}\right] \geq 0 \\
& \Longleftrightarrow\left[\begin{array}{cc}
P & \left(I-D_{k}\right) \\
\star & P^{-1}
\end{array}\right] \geq 0
\end{aligned}
$$

which, together with assumption (3.2) and Lemma 2.2, implies that

$$
P-\left(I-D_{k}\right)^{T} P\left(I-D_{k}\right) \geq 0 .
$$

Thus, it yields

$$
\begin{aligned}
V\left(t_{k}\right)= & \mu\left(t_{k}\right) y^{T}\left(t_{k}\right) P y\left(t_{k}\right)+\int_{t_{k}-\tau\left(t_{k}\right)}^{t_{k}} \mu(s) g^{T}(y(s)) Q g(y(s)) d s \\
& +\sum_{j=1}^{n} m_{j} \int_{0}^{\infty} h_{j}(\sigma) \int_{t_{k}-\sigma}^{t_{k}} \mu(s+\sigma) g_{j}^{2}\left(y_{j}(s)\right) d s d \sigma \\
= & \mu\left(t_{k}^{-}\right) y^{T}\left(t_{k}^{-}\right)\left(I-D_{k}\right)^{T} P\left(I-D_{k}\right) y\left(t_{k}^{-}\right) \\
& +\int_{t_{k}^{-}-\tau\left(t_{k}^{-}\right)}^{t_{k}^{-}} \mu(s) g^{T}(y(s)) Q g(y(s)) d s \\
& +\sum_{j=1}^{n} m_{j} \int_{0}^{\infty} h_{j}(\sigma) \int_{t_{k}^{-}-\sigma}^{t_{k}^{-}} \mu(s+\sigma) g_{j}^{2}\left(y_{j}(s)\right) d s d \sigma \\
\leq & \mu\left(t_{k}^{-}\right) y^{T}\left(t_{k}^{-}\right) P y\left(t_{k}^{-}\right)+\int_{t_{k}^{-}-\tau\left(t_{k}^{-}\right)}^{t_{k}^{-}} \mu(s) g^{T}(y(s)) Q g(y(s)) d s \\
& +\sum_{j=1}^{n} m_{j} \int_{0}^{\infty} h_{j}(\sigma) \int_{t_{k}^{-}-\sigma}^{t_{k}^{-}} \mu(s+\sigma) g_{j}^{2}\left(y_{j}(s)\right) d s d \sigma \\
\leq & V\left(t_{k}^{-}\right) .
\end{aligned}
$$


Hence, we can deduce that

$$
V\left(t_{k}\right) \leq V\left(t_{k}^{-}\right), \quad k \in \mathbb{Z}_{+}
$$

By (3.10) and (3.14), we know that $V$ is monotonically nonincreasing for $t \in[T, \infty)$, which implies that

$$
V(t) \leq V(T), \quad t \geq T
$$

It follows from the definition of $V$ that

$$
\mu(t) \lambda_{\min }(P)\|y(t)\|^{2} \leq \mu(t) y^{T}(t) P y(t) \leq V(t) \leq V_{0}<\infty, \quad t \geq 0,
$$

where $V_{0}=\max _{0 \leq s \leq T} V(s)$.

It implies that

$$
\|y(t)\|^{2} \leq \frac{V_{0}}{\mu(t) \lambda_{\min }(P)}, \quad t \geq 0
$$

This completes the proof of Theorem 3.1.

Remark 3.2. Theorem 3.1 provides a $\mu$-stability criterion for an impulsive differential system (2.5). It should be noted that the conditions in the theorem are dependent on the upper bound of the derivative of time-varying delay and the delay kernels $h_{j}, j \in$ $\Lambda$, and independent of the range of time-varying delay. Thus, it can be applied to impulsive neural networks with unbounded time-varying and continuously distributed delays.

Remark 3.3. In $[23,24]$, the authors have studied $\mu$-stability for neural networks with unbounded time-varying delays and continuously distributed delays via different approaches. However, the impulsive effect is not taken into account. Hence, our developed result in this paper complements and improves those reported in $[23,24]$. In particular, if we take $D_{k}=\operatorname{diag}\left(d_{1}^{(k)}, \ldots, d_{n}^{(k)}\right), d_{i}^{(k)} \in[0,2], i \in \Lambda, k \in \mathbb{Z}_{+}$, then the following result can be obtained.

Corollary 3.4. Assume that assumptions $\left(H_{1}\right),\left(H_{2}\right)$ and $\left(H_{3}\right)$ hold. Then, the zero solution of system (2.5) is $\mu$-stable if there exist some constants $\beta_{1} \geq 0, \beta_{2}>0, \beta_{3}>0, j \in \Lambda$, two $n \times n$ matrices $P>0, Q>0$, two diagonal positive definite $n \times n$ matrices $M=\operatorname{diag}\left(m_{1}, \ldots, m_{n}\right), U$, 
a nonnegative continuous differential function $\mu(t)$ defined on $[0, \infty)$, and a constant $T>0$ such that, for $t \geq T$

$$
\frac{\dot{\mu}(t)}{\mu(t)} \leq \beta_{1}, \quad \frac{\mu(t-\tau(t))}{\mu(t)} \geq \beta_{2}, \quad \frac{\int_{0}^{\infty} h_{j}(s) \mu(s+t) d s}{\mu(t)} \leq \beta_{3}, \quad j \in \Lambda,
$$

and the following LMIs hold:

$$
\left[\begin{array}{cccc}
\Sigma & P A+U \Sigma_{2} & P B & P W \\
\star & Q+N-U & 0 & 0 \\
\star & \star & -\beta_{2} Q(1-\rho) & 0 \\
\star & \star & \star & -M
\end{array}\right] \leq 0,
$$

where $\Sigma=\beta_{1} P-P C-C P-U \Sigma_{1}, N=\operatorname{diag}\left(m_{1} \beta_{3}, \ldots, m_{n} \beta_{3}\right)$.

If we take $\mu(t)=\mu$ ( $\mu$ denotes a constant), then the following global bounded result can be obtained.

Corollary 3.5. Assume that assumptions $\left(\mathrm{H}_{1}\right),\left(\mathrm{H}_{2}\right)$, and $\left(\mathrm{H}_{3}\right)$ hold. Then, the all solutions of system (2.5) have global boundedness if there exist two $n \times n$ matrices $P>0, Q>0$, two diagonal positive definite $n \times n$ matrices $M=\operatorname{diag}\left(m_{1}, \ldots, m_{n}\right), U$, such that, the following LMIs hold:

$$
\begin{gathered}
{\left[\begin{array}{cccc}
\Sigma & P A+U \Sigma_{2} & P B & P W \\
\star & Q+M-U & 0 & 0 \\
\star & \star & -Q(1-\rho) & 0 \\
\star & \star & \star & -M
\end{array}\right] \leq 0,} \\
{\left[\begin{array}{cc}
P & \left(I-D_{k}\right) P \\
\star & P
\end{array}\right] \geq 0,}
\end{gathered}
$$

where $\Sigma=-P C-C P-U$.

Remark 3.6. Notice that $\beta_{1}=0, \beta_{2}=1, \beta_{3}=1, j \in \Lambda$, and using the similar proof of Theorem 3.1, we can obtain the result easily.

\section{A Numerical Example}

In the following, we give an example to illustrate the validity of our method. 
Example 4.1. Consider a two-dimensional impulsive neural network with unbounded timevarying delays and continuously distributed delays:

$$
\begin{aligned}
\left(\begin{array}{l}
\dot{y}_{1}(t) \\
\dot{y}_{2}(t)
\end{array}\right)= & -\left(\begin{array}{ll}
3 & 0 \\
0 & 3
\end{array}\right)\left(\begin{array}{l}
y_{1}(t) \\
y_{2}(t)
\end{array}\right)+\left(\begin{array}{ll}
0.1 & 0.1 \\
0.1 & 0.1
\end{array}\right)\left(\begin{array}{l}
\tanh \left(y_{1}(t)\right) \\
\tanh \left(y_{2}(t)\right)
\end{array}\right) \\
& +\left(\begin{array}{cc}
0.1 & 0.1 \\
0.5 & -0.1
\end{array}\right)\left(\begin{array}{l}
\tanh \left(y_{1}(t-0.5 t)\right) \\
\tanh \left(y_{2}(t-0.5 t)\right)
\end{array}\right) \\
& +\left(\begin{array}{cc}
0.5 & 0.5 \\
0.5 & -0.5
\end{array}\right)\left(\begin{array}{l}
\int_{0}^{\infty} e^{-s} \tanh \left(y_{1}(t-s)\right) d s \\
\int_{0}^{\infty} e^{-s} \tanh \left(y_{2}(t-s)\right) d s
\end{array}\right), \quad t \neq t_{k}, t>0, \\
\left(\begin{array}{l}
\Delta y_{1}\left(t_{k}\right) \\
\Delta y_{2}\left(t_{k}\right)
\end{array}\right) & =-\left(\begin{array}{cc}
1.5 & 0 \\
0 & 1.5
\end{array}\right)\left(\begin{array}{l}
y_{1}\left(t_{k}^{-}\right) \\
y_{2}\left(t_{k}^{-}\right)
\end{array}\right), \quad t_{k}=k, k \in \mathbb{Z}_{+} .
\end{aligned}
$$

Then, $\tau(t)=0.5 t, h_{j}(s)=e^{-s}, \Sigma_{1}=\operatorname{diag}(0,0), \Sigma_{2}=\operatorname{diag}(0.5,0.5)$, and $\rho=0.5$. It is obvious that $(0,0)^{T}$ is an equilibrium point of system (4.1). Let $\mu(t)=t$ and choose $\beta_{1}=0.1$, $\beta_{2}=0.5, \beta_{3}=1.2$, then the LMIs in Theorem 3.1 have the following feasible solution via MATLAB LMI toolbox:

$$
\begin{array}{cl}
P=\left(\begin{array}{cc}
4.4469 & -0.0230 \\
-0.0230 & 4.3377
\end{array}\right), \quad Q=\left(\begin{array}{cc}
5.6557 & -0.2109 \\
-0.2109 & 5.5839
\end{array}\right), \\
M=\left(\begin{array}{cc}
5.5189 & 0 \\
0 & 5.5189
\end{array}\right), \quad U=\left(\begin{array}{cc}
20.5095 & 0 \\
0 & 20.5095
\end{array}\right) .
\end{array}
$$

The above results shows that all the conditions stated in Theorem 3.1 have been satisfied and hence system (4.1) with unbounded time-varying delay and continuously distributed delay is $\mu$-stable. The numerical simulations are shown in Figure 1.

\section{Conclusion}

In this paper, some sufficient conditions for $\mu$-stability of impulsive neural networks with unbounded time-varying delays and continuously distributed delays are derived. The results are described in terms of LMIs, which can be easily checked by resorting to available software packages. A numerical example has been given to demonstrate the effectiveness of the results obtained. 


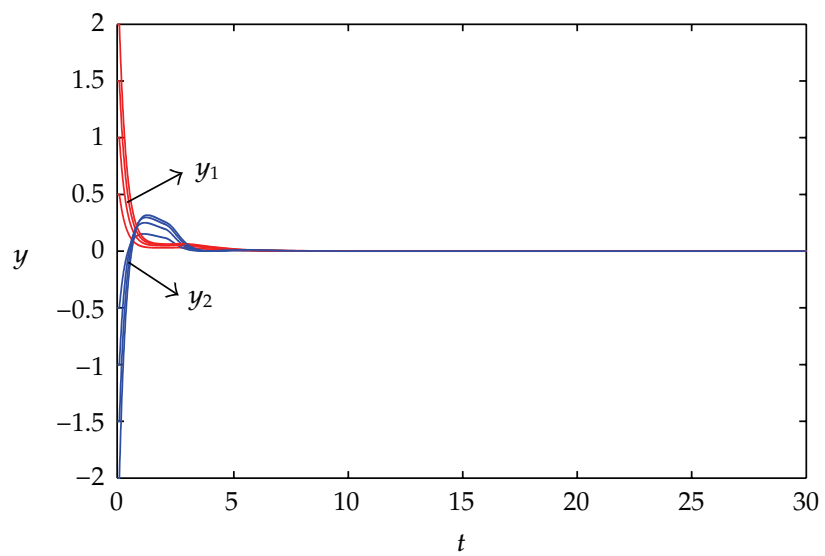

(a)

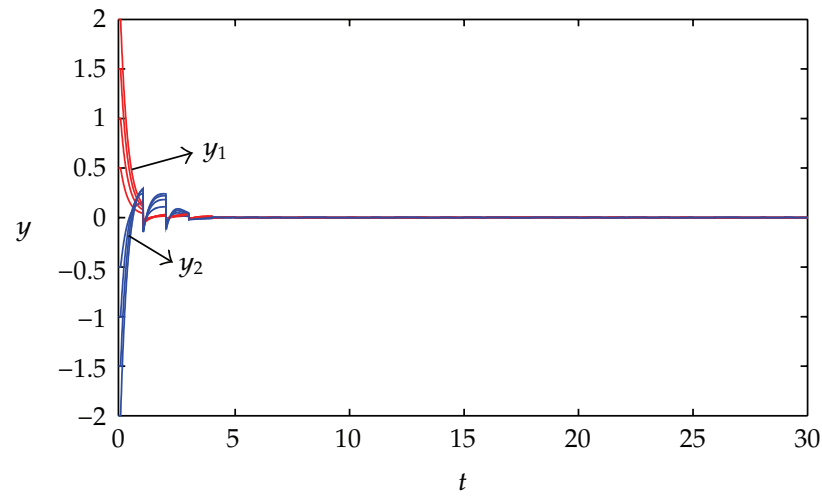

(b)

Figure 1: (a) State trajectories of system (4.1) without impulsive effects. (b) State trajectories of system (4.1) under impulsive effects.

\section{Acknowledgments}

This paper is supported by the National Natural Science Foundation of China (11071276), the Natural Science Foundation of Shandong Province (Y2008A29, ZR2010AL016), and the Science and Technology Programs of Shandong Province (2008GG30009008).

\section{References}

[1] L. O. Chua and L. Yang, "Cellular neural networks: theory," IEEE Transactions on Circuits and Systems, vol. 35, no. 10, pp. 1257-1272, 1988.

[2] M. A. Cohen and S. Grossberg, "Absolute stability of global pattern formation and parallel memory storage by competitive neural networks," IEEE Transactions on Systems, Man, and Cybernetics, vol. 13, no. 5, pp. 815-826, 1983.

[3] J. J. Hopfield, "Neurons with graded response have collective computational properties like those of two-state neurons," Proceedings of the National Academy of Sciences of the United States of America, vol. 81, no. 10 I, pp. 3088-3092, 1984.

[4] B. Kosko, "Bidirectional associative memories," IEEE Transactions on Systems, Man, and Cybernetics, vol. 18, no. 1, pp. 49-60, 1988. 
[5] Q. Zhang, X. Wei, and J. Xu, "Delay-dependent global stability results for delayed Hopfield neural networks," Chaos, Solitons \& Fractals, vol. 34, no. 2, pp. 662-668, 2007.

[6] S. Mohamad, K. Gopalsamy, and H. Akça, "Exponential stability of artificial neural networks with distributed delays and large impulses," Nonlinear Analysis: Real World Applications, vol. 9, no. 3, pp. 872-888, 2008.

[7] Q. Wang and X. Liu, "Exponential stability of impulsive cellular neural networks with time delay via Lyapunov functionals," Applied Mathematics and Computation, vol. 194, no. 1, pp. 186-198, 2007.

[8] Z.-T. Huang, Q.-G. Yang, and X. Luo, "Exponential stability of impulsive neural networks with timevarying delays," Chaos, Solitons \& Fractals, vol. 35, no. 4, pp. 770-780, 2008.

[9] X. Y. Lou and B. Cui, "New LMI conditions for delay-dependent asymptotic stability of delayed Hopfield neural networks," Neurocomputing, vol. 69, no. 16-18, pp. 2374-2378, 2006.

[10] V. Singh, "On global robust stability of interval Hopfield neural networks with delay," Chaos, Solitons E Fractals, vol. 33, no. 4, pp. 1183-1188, 2007.

[11] S. Arik, "Global asymptotic stability of hybrid bidirectional associative memory neural networks with time delays," Physics Letters, Section A, vol. 351, no. 1-2, pp. 85-91, 2006.

[12] Y. Zhang and J. Sun, "Stability of impulsive neural networks with time delays," Physics Letters, Section A, vol. 348, no. 1-2, pp. 44-50, 2005.

[13] X. Liao and C. Li, "An LMI approach to asymptotical stability of multi-delayed neural networks," Physica D, vol. 200, no. 1-2, pp. 139-155, 2005.

[14] S. Mohamad, "Exponential stability in Hopfield-type neural networks with impulses," Chaos, Solitons E Fractals, vol. 32, no. 2, pp. 456-467, 2007.

[15] O. Ou, "Global robust exponential stability of delayed neural networks: an LMI approach," Chaos, Solitons \& Fractals, vol. 32, no. 5, pp. 1742-1748, 2007.

[16] R. Rakkiyappan, P. Balasubramaniam, and J. Cao, "Global exponential stability results for neutraltype impulsive neural networks," Nonlinear Analysis: Real World Applications, vol. 11, no. 1, pp. 122 $130,2010$.

[17] R. Rakkiyappan and P. Balasubramaniam, “On exponential stability results for fuzzy impulsive neural networks," Fuzzy Sets and Systems, vol. 161, no. 13, pp. 1823-1835, 2010.

[18] R. Raja, R. Sakthivel, and S. M. Anthoni, "Stability analysis for discrete-time stochastic neural networks with mixed time delays and impulsive effects," Canadian Journal of Physics, vol. 88, no. 12, pp. 885-898, 2010.

[19] R. Sakthivel, R. Samidurai, and S. M. Anthoni, "New exponential stability criteria for stochastic BAM neural networks with impulses," Physica Scripta, vol. 82, no. 4, Article ID 045802, 2010.

[20] R. Sakthivel, R. Samidurai, and S. M. Anthoni, "Asymptotic stability of stochastic delayed recurrent neural networks with impulsive effects," Journal of Optimization Theory and Applications, vol. 147, no. 3, pp. 583-596, 2010.

[21] S.-I. Niculescu, Delay Effects on Stability: A RobustControl Approach, vol. 269 of Lecture Notes in Control and Information Sciences, Springer, London, UK, 2001.

[22] V. B. Kolmanovskir and V. R. Nosov, Stability of Functional Differential Equations, vol. 180 of Mathematics in Science and Engineering, Academic Press, London, UK, 1986.

[23] T. Chen and L. Wang, "Global $\mu$-stability of delayed neural networks with unbounded time-varying delays," IEEE Transactions on Neural Networks, vol. 18, no. 6, pp. 705-709, 2007.

[24] X. Liu and T. Chen, "Robust $\mu$-stability for uncertain stochastic neural networks with unbounded time-varying delays," Physica A, vol. 387, no. 12, pp. 2952-2962, 2008.

[25] V. Lakshmikantham, D. D. Baĭnov, and P. S. Simeonov, Theory of Impulsive Differential Equations, vol. 6 of Series in Modern Applied Mathematics, World Scientific, Teaneck, NJ, USA, 1989.

[26] D. D. Baĭnov and P. S. Simeonov, Systems with Impulse Effect: Stability Theory and Applications, Ellis Horwood Series: Mathematics and Its Applications, Ellis Horwood, Chichester, UK, 1989.

[27] X. Li, "Uniform asymptotic stability and global stabiliy of impulsive infinite delay differential equations," Nonlinear Analysis: Theory, Methods E Applications, vol. 70, no. 5, pp. 1975-1983, 2009.

[28] X. Li, X. Fu, P. Balasubramaniam, and R. Rakkiyappan, "Existence, uniqueness and stability analysis of recurrent neural networks with time delay in the leakage term under impulsive perturbations," Nonlinear Analysis: Real World Applications, vol. 11, no. 5, pp. 4092-4108, 2010.

[29] S. Boyd, L. El Ghaoui, E. Feron, and V. Balakrishnan, Linear Matrix Inequalities in System and Control Theory, vol. 15 of SIAM Studies in Applied Mathematics, Society for Industrial and Applied Mathematics (SIAM), Philadelphia, Pa, USA, 1994. 